\title{
Streamline topologies near simple degenerate critical points in two-dimensional flow away from boundaries
}

Brøns, Morten; Hartnack, Johan Nicolai

Published in:

Physics of Fluids

Link to article, DOI:

10.1063/1.869881

Publication date:

1999

Document Version

Publisher's PDF, also known as Version of record

Link back to DTU Orbit

Citation (APA):

Brøns, M., \& Hartnack, J. N. (1999). Streamline topologies near simple degenerate critical points in two-

dimensional flow away from boundaries. Physics of Fluids, 11(2), 314-324. https://doi.org/10.1063/1.869881

\section{General rights}

Copyright and moral rights for the publications made accessible in the public portal are retained by the authors and/or other copyright owners and it is a condition of accessing publications that users recognise and abide by the legal requirements associated with these rights.

- Users may download and print one copy of any publication from the public portal for the purpose of private study or research.

- You may not further distribute the material or use it for any profit-making activity or commercial gain

- You may freely distribute the URL identifying the publication in the public portal

If you believe that this document breaches copyright please contact us providing details, and we will remove access to the work immediately and investigate your claim. 


\title{
Streamline topologies near simple degenerate critical points in two-dimensional flow away from boundaries
}

\author{
Morten Brøns and Johan Nicolai Hartnack \\ Department of Mathematics, Technical University of Denmark, Building 303, DK-2800 Lyngby, Denmark
}

(Received 16 April 1998; accepted 23 October 1998)

\begin{abstract}
Streamline patterns and their bifurcations in two-dimensional incompressible flow are investigated from a topological point of view. The velocity field is expanded at a point in the fluid, and the expansion coefficients are considered as bifurcation parameters. A series of nonlinear coordinate changes results in a much simplified system of differential equations for the streamlines (a normal form) encapsulating all the features of the original system. From this, we obtain a complete description of bifurcations up to codimension three close to a simple linear degeneracy. As a special case we develop the theory for flows with reflectional symmetry. We show that all the patterns obtained can be realized in steady Navier-Stokes or Stokes flow, but an unresolved difficulty arises in the symmetric case for Navier-Stokes flow. The theory is applied to the patterns and bifurcations found numerically in two recent studies of Stokes flow in confined domains. (C) 1999 American Institute of Physics. [S1070-6631(99)02102-9]
\end{abstract}

\section{INTRODUCTION}

A number of fundamental concepts used to describe fluid flows involve local properties of the streamlines. Think, for example, of vortices and eddies, stagnation points, dividing streamlines, recirculating zones, and the process of separation. Even if some of these phenomena do not have an exact definition in terms of streamlines (and even no formal definition at all ${ }^{1}$ ), streamline patterns always play a role in identifying important properties of a flow. In the present paper we examine streamline patterns and their bifurcations from a topological point of view, using methods from nonlinear dynamics.

Given a velocity field $\mathbf{v}(\mathbf{x})$ at some time instant $t_{0}$, the streamlines are found as trajectories of the ordinary differential equations $\dot{\mathbf{x}}=\mathbf{v}(\mathbf{x})$, a nonlinear dynamical system in dimension two or three. Low-dimensional nonlinear dynamics is concerned exactly with the topology of the trajectories of such equations and hence provides a coherent theoretical framework as well as a terminology for fluid structures. For example, a stagnation point (where $\mathbf{v}=\mathbf{0}$ ) is known in dynamics as a critical point, and for two-dimensional (2-D) incompressible flow there are two nondegenerate possibilities: If the point is a center, the fluid mechanics interpretation is a vortex or eddying motion, while a saddle represents a point of stagnation where the separatrices are the dividing streamlines.

The qualitative study of streamlines using tools and ideas from nonlinear dynamics has a long and scattered history in fluid mechanics; see, e.g., the review papers. ${ }^{2,3}$ Early work on separation was performed by Legendre, ${ }^{4}$ Oswatitsch, ${ }^{5}$ Davey, ${ }^{6}$ and Lighthill. ${ }^{7}$ Hunt et al. ${ }^{8}$ studied flow around obstacles and Dallmann ${ }^{9-11}$ have considered both separation and vortex structures. Other applications are wake flow behind bluff bodies, ${ }^{12-14}$ flow close to free and viscous surfaces, ${ }^{15,16}$ channel flow over a step, ${ }^{17}$ axisymmet- ric flow, ${ }^{18,19}$ and the streamline topology of point vortex flow. ${ }^{20}$ In visualization experiments a topological approach has been used for pattern recognition ${ }^{21}$ and for constructing a wavelet basis for structure identification. ${ }^{22}$

When the flow field changes due to unsteadiness of the flow or an external change of parameters or boundary conditions, the streamline topology may also change. When the flow field is varied through a degenerate configuration that is structurally unstable, the streamline pattern can bifurcate and new structures arise. Such bifurcations for 2-D flow close to a planar wall and in conical flow are studied by Bakker. ${ }^{23}$ Our aim in the present study is the same, but for 2-D flow away from boundaries.

To obtain local information about a velocity field close to a critical point, we use a Taylor expansion of the streamfunction. The coefficients in the Taylor series are then considered as bifurcation parameters. Even for low-order expansions, the number of coefficients is large, and the bifurcation analysis is complicated. However, by a systematic use of normal form transformations, ${ }^{24}$ the number of free parameters is reduced significantly. This technique was used by Brøns ${ }^{18}$ for axisymmetric flow and by Hartnack ${ }^{25}$ to describe flow close to fixed, possibly curved, walls.

We will classify the possible degenerate patterns that occur with a simple degeneracy in the linear part and then obtain their unfoldings, that is, all possible patterns that can occur when such a configuration is perturbed slightly. A degeneracy is characterized by its codimension, which is the minimal number of parameters needed to describe the unfolding. From the normal form analysis the codimension is readily determined, and we show the associated bifurcation diagrams of codimension up to three. As a special case we develop the theory for flow with reflectional symmetry.

We also show that the constraints imposed by the vorticity transport equation for steady Navier-Stokes or Stokes flow has no consequence for the streamline topology in the 
general case. In the symmetrical case the same holds for Stokes flow, but a structural difference leaves the problem open for Navier-Stokes flow.

Our main purpose in this paper is to establish a theoretical framework for analyzing flow topologies and their metamorphoses. To illustrate its scope we conclude by indicating how the theory can be applied to understand the streamline patterns and bifurcations recently observed numerically in two specific Stokes flows: the flow in a rectangular cavity ${ }^{26}$ and the flow between rotating coaxial cylinders. ${ }^{27}$

\section{GENERAL FLOW PATTERNS}

\section{A. Taylor expansions and regular critical points}

We consider an incompressible two-dimensional flow far from any boundaries. A streamfunction $\psi$ exists such that the streamlines are found from

$$
\dot{x}=u=\frac{\partial \psi}{\partial y}, \quad \dot{y}=v=-\frac{\partial \psi}{\partial x} .
$$

To obtain local information of the flow close to a given point that we take to be the origin, $\psi$ is expanded in a power series,

$$
\psi=\sum_{i+j=1}^{\infty} a_{i, j} x^{i} y^{j}
$$

For the arguments in this section, the analyticity of $\psi$ is not important. We will impose certain nondegeneracy conditions on the coefficients at some finite order, and the results we obtain only depend on $\psi$ being continuously differentiable to that order.

The expansion coefficients in Eq. (2) have a direct physical interpretation. The first-order coefficients are related to the components of the velocity vector at the origin,

$$
a_{0,1}=u(0,0), \quad a_{1,0}=-v(0,0),
$$

and the higher-order coefficients are related to the viscous stress tensor and its derivatives evaluated at the origin. For example,

$$
\begin{aligned}
& \tau_{x x}=-\tau_{y y}=2 \mu \frac{\partial u}{\partial x}=2 \mu a_{1,1}, \\
& \tau_{x y}=\tau_{y x}=\mu\left(\frac{\partial u}{\partial y}+\frac{\partial v}{\partial x}\right)=2 \mu\left(a_{0,2}-a_{2,0}\right) .
\end{aligned}
$$

The linear approximation of the equations for the streamlines is

$$
\left(\begin{array}{c}
\dot{x} \\
\dot{y}
\end{array}\right)=\left(\begin{array}{c}
a_{0,1} \\
-a_{1,0}
\end{array}\right)+\left(\begin{array}{cc}
a_{1,1} & 2 a_{0,2} \\
-2 a_{2,0} & -a_{1,1}
\end{array}\right)\left(\begin{array}{l}
x \\
y
\end{array}\right) .
$$

If $a_{1,0}=a_{0,1}=0$ the origin is a critical point. The streamline pattern close to the origin is determined using standard theory for Hamiltonian systems. If the determinant of the Jacobian matrix $|J|=4 a_{2,0} a_{0,2}-a_{1,1}^{2}$ is positive, the critical point is a center, if $|J|$ is negative it is a saddle. See Fig. 1. In terms of physical quantities, we have

$$
|J|=4 a_{2,0} a_{0,2}-a_{1,1}^{2}=-\frac{\partial u}{\partial y} \frac{\partial v}{\partial x}+\frac{\partial u}{\partial x} \frac{\partial v}{\partial y}=\widehat{\nabla u} \cdot \nabla v,
$$

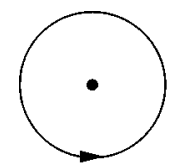

(a)

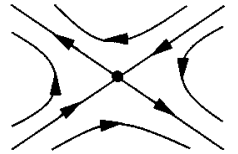

(b)
FIG. 1. Nondegenerate critical points. (a) Center; (b) saddle.

showing that the type of the critical point depends on the angle between the gradients of the velocity components at the point.

\section{B. Degenerate critical points and normal forms}

If $|J|=0$, the critical point is degenerate, and higherorder terms become decisive for the streamline pattern. There are two distinct subcases. Either zero is an eigenvalue with geometric multiplicity one, or $J$ is the zero matrix. In the present paper we consider the first case only, which we will refer to as a simple linear degeneracy. The latter case is considerably more difficult and we briefly touch upon it in Sec. IV and Sec. VI. In terms of physical quantities we have

$$
\widehat{\nabla} u \cdot \nabla v=0, \quad \text { with }|\boldsymbol{\nabla} u|+|\boldsymbol{\nabla} v| \neq 0 .
$$

Hence, in a simple linear degenerate critical point the gradients of $u$ and $v$ are parallel or at most one of them is the zero vector.

Without loss of generality, we can assume that the coordinate system is chosen such that

$$
a_{1,1}=a_{2,0}=0, \text { but } a_{0,2} \neq 0 \text {. }
$$

Before analyzing the system (1) we will make a series of normal form transformations ${ }^{24}$ to successively simplify quadratic, cubic, and higher-order terms. The system is Hamiltonian and we wish to preserve this property under the transformation. Among all transformations preserving the Hamiltonian structure canonical transformations have been widely used in analytical mechanics. For a one-degree-offreedom system as considered here, a canonical transformation is simply an area-preserving map of the phase space (the physical flow plane).

Canonical transformations are easily found via generating functions. Denote the new coordinates $(\xi, \eta)$. For a smooth function $S(y, \xi)$ consider the equations

$$
x=\frac{\partial S}{\partial y}, \quad \eta=\frac{\partial S}{\partial \xi} .
$$

If these can be solved for $(x, y)$, the solution defines a canonical transformation from new to old coordinates, $x=x(\xi, \eta)$, $y=y(\xi, \eta)$ (Ref. 28, Chap. 8).

To simplify the quadratic terms of Eqs. (1)—cubic terms of $\psi$-the normal form procedure uses a transformation that is the identity plus quadratic terms, and, possibly, higherorder terms as well. We choose a generating function,

$$
S=y \xi+\sum_{i+j=3} s_{i, j} y^{i} \xi^{j} .
$$




$$
\begin{gathered}
x=\xi+3 s_{3,0} y^{2}+2 s_{2,1} y \xi+s_{1,2} \xi^{2}, \\
\eta=y+s_{2,1} y^{2}+2 s_{1,2} y \xi+3 s_{0,3} \xi^{2} .
\end{gathered}
$$

We look for solutions to Eqs. (11) in the form of Taylor series,

$$
x=\xi+\sum_{i+j=2}^{\infty} \alpha_{i, j} \xi^{i} \eta^{j}, \quad y=\eta+\sum_{i+j=2}^{\infty} \beta_{i, j} \xi^{i} \eta^{j} .
$$

Inserting these in Eqs. (11) and collecting terms to the same order in $\xi, \eta$, linear equations for the Taylor coefficients $\alpha_{i, j}, \beta_{i, j}$ are obtained. For the second-order coefficients the solutions are

$$
\begin{aligned}
& \alpha_{2,0}=s_{1,2}, \quad \alpha_{1,1}=2 s_{2,1}, \quad \alpha_{0,2}=3 s_{3,0}, \\
& \beta_{2,0}=-3 s_{0,3}, \quad \beta_{1,1}=-2 s_{1,2}, \quad \beta_{0,2}=-s_{2,1} .
\end{aligned}
$$

Hence, the transformation (12) becomes

$$
\begin{aligned}
& x=\xi+s_{1,2} \xi^{2}+2 s_{2,1} \xi \eta+3 s_{3,0} \eta^{2}+\mathcal{O}\left[|(\xi, \eta)|^{3}\right], \\
& y=\eta-3 s_{0,3} \xi^{2}-2 s_{1,2} \xi \eta-s_{2,1} \eta^{2}+\mathcal{O}\left[|(\xi, \eta)|^{3}\right] .
\end{aligned}
$$

Inserting this transformation in Eq. (2), we obtain

$$
\begin{aligned}
\psi= & a_{0,2} \eta^{2}+a_{3,0} \xi^{3}+\left(a_{2,1}-6 a_{0,2} s_{0,3}\right) \xi^{2} \eta \\
& +\left(a_{1,2}-4 a_{0,2} s_{1,2}\right) \xi \eta^{2}+\left(a_{0,3}-2 a_{0,2} s_{2,1}\right) \eta^{3} \\
& +\mathcal{O}\left[|(\xi, \eta)|^{4}\right] .
\end{aligned}
$$

The $s_{i, j}$ are free for us to choose. First, we see that $s_{3,0}$ does not appear in Eq. (14) and we arbitrarily set it to zero. Second, with the choices

$$
s_{2,1}=\frac{a_{0,3}}{2 a_{0,2}}, \quad s_{1,2}=\frac{a_{1,2}}{4 a_{0,2}}, \quad s_{0,3}=\frac{a_{2,1}}{6 a_{0,2}},
$$

we eliminate a number of terms and get

$$
\psi=a_{0,2} \eta^{2}+a_{3,0} \xi^{3}+\mathcal{O}\left[|(\xi, \eta)|^{4}\right] .
$$

If $a_{3,0} \neq 0$, the only remaining cubic term of $\psi$ is nondegenerate, and, as we shall see below, the streamline pattern can be determined. If $a_{3,0}=0$, the critical point is nonlinear degenerate to order three, and further terms in the expansion of $\psi$ must be computed.

To continue and simplify the quartic terms of $\psi$, we use a generating function of the form

$$
S=y \xi+\sum_{i+j=3} s_{i, j} y^{i} \xi^{j}+\sum_{i+j=4} s_{i, j} y^{i} \xi^{j},
$$

where the $s_{i, j}$ of order three are given by (15) and the $s_{i, j}$ of order four now are free parameters. The Taylor series of the transformation (12) must be determined to order three in the same way as above, and by insertion in $\psi$, the $s_{i, j}$ are to be chosen to eliminate as many terms as possible.

In the general case, to any finite order $N$, one can show that it is possible to choose the transformation, such that

$$
\psi=a_{0,2} \eta^{2}+\sum_{i=3}^{N} \tilde{a}_{i, 0} \xi^{i}+\mathcal{O}\left[|(\xi, \eta)|^{N+1}\right],
$$

where

$$
\widetilde{a}_{3,0}=a_{3,0},
$$

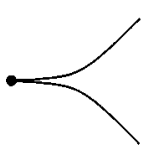

(a)

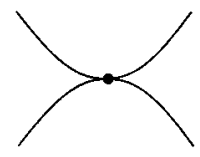

(b)

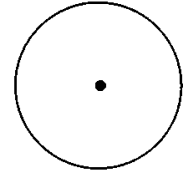

(c)
FIG. 2. Degenerate critical points in the flow field. (a) Cusp. (b) Topological saddle. (c) Center.

$$
\begin{aligned}
& \widetilde{a}_{4,0}=a_{4,0}-\frac{a_{2,1}^{2}}{4 a_{0,2}}+\mathcal{O}\left(a_{3,0}\right), \\
& \tilde{a}_{5,0}=a_{5,0}+a_{4,0} \frac{a_{1,2}}{a_{0,2}}-a_{3,1} \frac{a_{2,1}}{2 a_{0,2}}+\mathcal{O}\left(a_{3,0}\right),
\end{aligned}
$$

etc. In $\widetilde{a}_{k, 0}$, the term $\mathcal{O}\left(a_{3,0}\right)$ denotes expressions linear in $a_{3,0}$ and also depending on $a_{i, j}$ with $i+j<k$. Hence, $\widetilde{a}_{k, 0}$ is $a_{k, 0}$ plus nonlinear combinations of $a_{i, j}$, where $i+j<k$. The source of the complexity of the expressions in Eqs. (19) is apparent from the coordinate transformations. The terms in the transformation to some order $n$ do not only modify terms of $\psi$ to the same order, but to higher orders as well. The exact expressions are readily computed, but they are long and complicated and shed no further light on the problem, as no physical interpretation presents itself.

The normal form of order $N$ is obtained by dropping the $\mathcal{O}$ term in Eq. (18). By the use of the normal form it is now very easy to find the local flow picture of the degenerate configurations. A degeneracy of order $N$ occurs if $\widetilde{a}_{3,0}$ $=\cdots=\widetilde{a}_{N-1,0}=0$, but $\widetilde{a}_{N, 0} \neq 0$. Then the normal form of $\psi$ is

$$
\psi=a_{0,2} \eta^{2}+\widetilde{a}_{N, 0} \xi^{N} \text {. }
$$

Possible separatrices (dividing streamlines) of the critical point are given by $\psi=0$, that is

$$
\eta= \pm \sqrt{-\frac{\widetilde{a}_{N, 0}}{a_{0,2}} \xi^{N}}
$$

If $N$ is odd this describes two streamlines that meet at the critical point in a cusp. If $N$ is even, two subcases occur: If $\widetilde{a}_{N, 0} / a_{0,2}>0$ there are no separatrices and the critical point is a degenerate center; if $\widetilde{a}_{N, 0} / a_{0,2}<0$ we obtain four separatrices, and this case is denoted a topological saddle. ${ }^{23}$ See Fig. 2.

\section{Unfoldings of degenerate critical points}

The patterns found in the previous section only occur when the parameters take certain degenerate values. A small change from these combinations will result in qualitatively different patterns. To study the bifurcations close to the simple linear degeneracy, we introduce small parameters,

$$
\epsilon_{1}=a_{1,0}, \quad \epsilon_{2}=a_{0,1}, \quad \epsilon_{3}=a_{2,0}, \quad \epsilon_{4}=a_{1,1},
$$

which all take the value zero when the origin is a critical point with a simple linear degeneracy.

We proceed as before, but now include the small parameters in the transformations. We briefly sketch how to simplify quadratic terms. We choose a generating function, 


$$
S=y \xi+\sum_{i+j+k+l+m+n=3} s_{i, j, k, l, m, n} y^{i} \xi^{j} \epsilon_{1}^{k} \epsilon_{2}^{l} \epsilon_{3}^{m} \epsilon_{4}^{n},
$$

and the new coordinates are found through the relations

$$
x=\frac{\partial S}{\partial y}, \quad \eta=\frac{\partial S}{\partial \xi} .
$$

Solving to find the transformation and inserting in the streamfunction, we obtain

$$
\begin{aligned}
\psi= & \mu_{1}+\mu_{2} \xi+\mu_{3} \eta+\mu_{4} \xi^{2}+\left(2 s_{2,1,0,0,0,0}-4 a_{0,2} s_{0,2,1,0,0,0}\right) \epsilon_{1} \xi \eta+\left(-2 s_{1,2,0,0,0,0}-4 a_{0,2} s_{0,2,0,1,0,0}\right) \epsilon_{2} \xi \eta \\
& -4 a_{0,2} s_{0,2,0,0,1,0} \epsilon_{3} \xi \eta+\left(1-4 a_{0,2} s_{0,2,0,0,1}\right) \epsilon_{4} \xi \eta+a_{3,0} \xi^{3}-\left(4 a_{0,2} s_{1,2,0,0,0,0}-a_{1,2}\right) \xi \eta^{2}+\left(-6 a_{0,2} s_{0,3,0,0,0,0}+a_{2,1}\right) \xi^{2} \eta \\
& +\left(-2 a_{0,2} s_{2,1,0,0,0,0}+a_{0,3}\right) \eta^{3}+\mathcal{O}(4),
\end{aligned}
$$

where the $\mu$ 's are new small parameters that are algebraic combinations of the original $\epsilon$ 's and $\mathcal{O}(4)$ denotes terms to order four in both coordinates and parameters. By choosing

$$
\begin{aligned}
& s_{1,2,0,0,0,0}=\frac{a_{1,2}}{4 a_{0,2}}, \quad s_{0,3,0,0,0,0}=\frac{a_{2,1}}{6 a_{0,2}}, \quad s_{2,1,0,0,0,0}=\frac{a_{0,3}}{2 a_{0,2}}, \\
& s_{0,2,0,0,0,1}=\frac{1}{4 a_{0,2}}, \quad s_{0,2,0,1,0,0}=-\frac{a_{1,2}}{8 a_{0,2}^{2}}, \\
& s_{0,2,1,0,0,0}=\frac{a_{0,3}}{4 a_{0,2}^{2}},
\end{aligned}
$$

and setting the remaining coefficients in the generating function $S$ equal to zero, the result is

$$
\begin{aligned}
\psi= & \left(\epsilon_{1}-\frac{\epsilon_{2} \epsilon_{4}}{2 a_{0,2}}+\frac{a_{1,2} \epsilon_{2}^{2}}{4 a_{0,2}^{2}}-\frac{a_{0,3} \epsilon_{1} \epsilon_{2}}{2 a_{0,2}^{2}}\right) \xi+\epsilon_{2} \eta \\
& +\left(\epsilon_{3}+\frac{a_{1,2} \epsilon_{1}}{4 a_{0,2}}-\frac{a_{2,1} \epsilon_{2}}{2 a_{0,2}}\right) \xi^{2}+\widetilde{a}_{0,2} \eta^{2}+a_{3,0} \xi^{3}+\mathcal{O}(4),
\end{aligned}
$$

with

$$
\tilde{a}_{0,2}=a_{0,2}-\frac{a_{0,3} \epsilon_{2}}{2 a_{0,2}} .
$$

Note that all mixed terms in $\xi$ and $\eta$ have been removed. If $a_{3,0} \neq 0$, a further simplification is possible. The term of the next-highest degree in a univariate polynomial can always be removed by a suitable translation of the origin. This is achieved by replacing $\xi$ by $\xi+\xi_{0}$ and $\eta$ by $\eta+\eta_{0}$, where

$$
\xi_{0}=\frac{2 a_{2,1} \epsilon_{2}-a_{1,2} \epsilon_{1}-4 \epsilon_{3} a_{0,2}}{12 a_{0,2} a_{3,0}}, \quad \eta_{0}=\frac{\epsilon_{2} a_{0,2}}{a_{0,3} \epsilon_{2}-2 a_{0,2}^{2}} .
$$

A final simplification can be obtained by first dividing $\psi$ by $2 \widetilde{a}_{0,2}-$ corresponding to scaling the time- and then scale $\xi$ by the substitution

$$
\xi \rightarrow\left(\frac{2 \widetilde{a}_{0,2}}{3 a_{3,0}}\right)^{1 / 3} \xi
$$

to obtain

$$
\psi=\frac{1}{2} \eta^{2}+c_{0}+c_{1} \xi+\frac{1}{3} \xi^{3}+\mathcal{O}(4),
$$

where $c_{0}, c_{1}$ are new small parameters depending on $\epsilon_{1}, \ldots, \epsilon_{4}$. Since the streamlines lie on isocurves of $\psi$ we can further omit the $c_{0}$ term.

If $a_{3,0}$ is also small, that is, we are close to a nonlinear degeneracy of order four or more, terms of higher order must be computed. In the general case one obtains, relabeling the coordinates back to $x, y$, the following.

Theorem II.1: Let $a_{1,0}, a_{0,1}, a_{2,0}, \quad a_{1,1}$, and $\widetilde{a}_{3,0}, \cdots, \widetilde{a}_{N-1,0}$ be small parameters. Assuming the nondegeneracy conditions $a_{0,2} \neq 0, \widetilde{a}_{N, 0} \neq 0$ a normal form of order $N$ for the streamfunction (2) is

$$
\begin{aligned}
& \psi_{N}=\frac{\sigma}{2} y^{2}+f(x), \quad f(x)=\sum_{i=1}^{N} c_{i} x^{i}, \quad c_{N-1}=0, \\
& c_{N}=\frac{1}{N},
\end{aligned}
$$

where

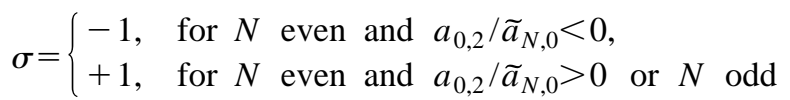

and $c_{i}, i=1, \ldots, N-2$ are transformed small parameters.

The two subcases for $N$ even stem from the scaling (29), which then is

$$
\xi \rightarrow\left|\frac{2 \widetilde{a}_{0,2}}{N \widetilde{a}_{N, 0}}\right|^{1 / N} \xi
$$

If a negative coefficient to the $\xi^{N}$ term occurs, $\psi$ is replaced by $-\psi$ and $\sigma=-1$ results.

Some general features of the normal form are evident. First, $\psi$ is an even function of $y$, and all resulting streamline patterns will have reflectional symmetry in the $x$ axis. The differential equations for the streamlines are

$$
\dot{x}=\sigma y, \quad \dot{y}=-f^{\prime}(x) .
$$

All critical points lie on the $x$ axis and are found as solutions to $f^{\prime}(x)=0$. That is, there are, at most, $N-1$ critical points. At a critical point, the Jacobian is

$$
\left(\begin{array}{cc}
0 & \sigma \\
-f^{\prime \prime}(x) & 0
\end{array}\right)
$$

and we immediately obtain that the critical point is a center if $\sigma f^{\prime \prime}(x)>0$ and a saddle if $\sigma f^{\prime \prime}(x)<0$. The vorticity is

$$
\omega=\nabla^{2} \psi_{N}=\sigma\left[\sigma f^{\prime \prime}(x)+1\right],
$$



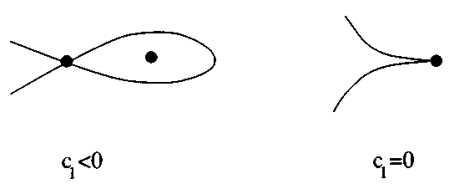

$c_{1}=0$

FIG. 3. Bifurcation diagram for the normal form of order 3, Eq. (38).

which always has the sign of $\sigma$ at a center. Thus, the vortices or eddying structures that occur all have the same sense of rotation.

If $f^{\prime \prime}(x)=0$, the critical point is degenerate and bifurcation occurs. The type of a degenerate point $\left(x_{c}, 0\right)$ can easily be found by expanding the $\psi$ in that point,

$$
\begin{aligned}
\psi= & \frac{\sigma}{2} y^{2}+f\left(x_{c}\right)+\frac{1}{6}\left(x-x_{c}\right)^{3} f^{\prime \prime \prime}\left(x_{c}\right) \\
& +\cdots+\frac{1}{n !}\left(x-x_{c}\right)^{n} f^{(n)}\left(x_{c}\right) .
\end{aligned}
$$

Separatrices are found as solutions to $\psi=f\left(x_{c}\right)$ and it follows that the type of the critical point depends on the first nonvanishing derivative of $f(x)$. That is, if the order of the first nonvanishing derivative is odd the degenerate point is a cusp and if the order is even the critical point is either a center $(\sigma=1)$ or a topological saddle $(\sigma=-1)$.

In addition to local bifurcations associated with degenerate critical points, there is also the possibility of global bifurcations. These occur at parameter values where critical points are connected by heteroclinic trajectories. This requires $\psi$ to attain the same value at the critical points. The algebraic condition for this is

$$
\begin{aligned}
& f\left(x_{1}\right)=f\left(x_{2}\right)=\cdots=f\left(x_{p}\right) \text { and } \\
& f^{\prime}\left(x_{1}\right)=f^{\prime}\left(x_{2}\right)=\cdots=f^{\prime}\left(x_{p}\right)=0,
\end{aligned}
$$

for all the $p$ critical points $\left(x_{i}, 0\right)$ on the heteroclinic cycle.

\section{Normal form of order 3}

For $N=3$, the streamfunction is

$$
\psi_{3}=\frac{1}{2} y^{2}+c_{1} x+\frac{1}{3} x^{3} .
$$

For $c_{1}=0$ the origin is a cusp. The critical points are found from

$$
f^{\prime}(x)=c_{1}+x^{2}=0,
$$

which has solutions if $c_{1}<0$ given by $x= \pm \sqrt{-c_{1}}$. Here we have $f^{\prime \prime}(x)=2 x$, showing that the two critical points are a saddle and a center. The unfolding is shown in Fig. 3. This is the simplest possible way critical points can be created, and this bifurcation occurs in a large variety of flow problems. We show some examples of this in Sec. V.

\section{Normal form of order 4}

For $N=4$, the streamfunction is

$$
\psi_{4}=\frac{\sigma}{2} y^{2}+c_{1} x+c_{2} x^{2}+\frac{1}{4} x^{4} .
$$

The condition for bifurcation is

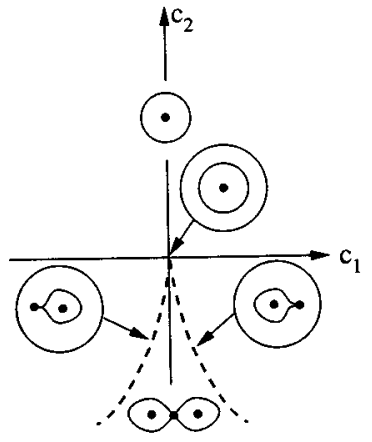

(a)

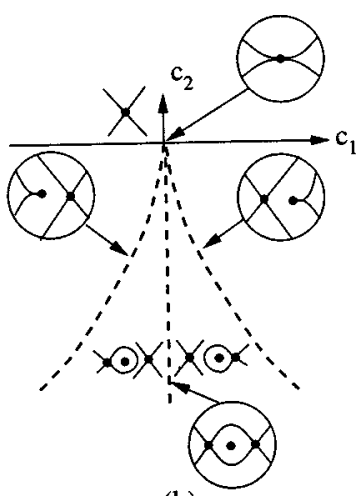

(b)
FIG. 4. Bifurcation diagram for the normal form of order 4, Eq. (40). (a) $\sigma=1$, (b) $\sigma=-1$.

$$
f^{\prime}(x)=c_{1}+2 c_{2} x+x^{3}=0, \quad f^{\prime \prime}(x)=2 c_{2}+3 x^{2}=0 .
$$

By eliminating $x= \pm \sqrt{-2 c_{2} / 3}$ we find that bifurcation occurs for

$$
32 c_{2}^{3}+27 c_{1}^{2}=0,
$$

describing a bifurcation curve in the $\left(c_{1}, c_{2}\right)$ parameter plane. At the degenerate critical points, we have $f^{\prime \prime \prime} \neq 0$, showing that they are cusps. Thus, as the bifurcation curve is crossed, two critical points are created or destroyed, as shown in Fig. 4. A simple computation shows that there is one critical point when $32 c_{2}^{3}+27 c_{1}^{2}>0$, and three critical points when $32 c_{2}^{3}+27 c_{1}^{2}<0$. Their type depend on $\sigma$.

For $\sigma=-1$, there can be two saddles, and a possibility for heteroclinic bifurcation. A straightforward computation shows that the conditions (37) are fulfilled when $c_{1}=0$, $c_{2}<0$. The process sketched in Fig. 4(a) can be viewed as an eddy obtaining an inner substructure.

\section{Normal form of order 5}

For $N=5$ the streamfunction becomes

$$
\psi_{5}=\frac{1}{2} y^{2}+c_{1} x+c_{2} x^{2}+c_{3} x^{3}+\frac{1}{5} x^{5} .
$$

For $c_{1}=c_{2}=c_{3}=0$ the origin is a cusp. Again, local bifurcations occur for

$$
\begin{aligned}
& f^{\prime}(x)=c_{1}+2 c_{2} x+3 c_{3} x^{2}+x^{4}=0, \\
& f^{\prime \prime}(x)=2 c_{2}+6 c_{3} x+4 x^{3}=0 .
\end{aligned}
$$

Combining these, one obtains

$$
x^{4}+c_{3} x^{2}-\frac{1}{3} c_{1}=0 .
$$

It is convenient to use the discriminant $D$ of this equation as a parameter rather than $c_{1}$,

$$
D=c_{3}^{2}+\frac{4}{3} c_{1} .
$$

When $D \geqslant 0$ local bifurcation occurs for

$$
c_{2}^{2}=\frac{1}{2}\left(-c_{3} \pm \sqrt{D}\right)\left(2 c_{3} \pm \sqrt{D}\right)^{2},
$$

for $D<0$ no local bifurcation occurs. The conditions for global bifurcation are

$$
f\left(x_{1}\right)=f\left(x_{2}\right),
$$




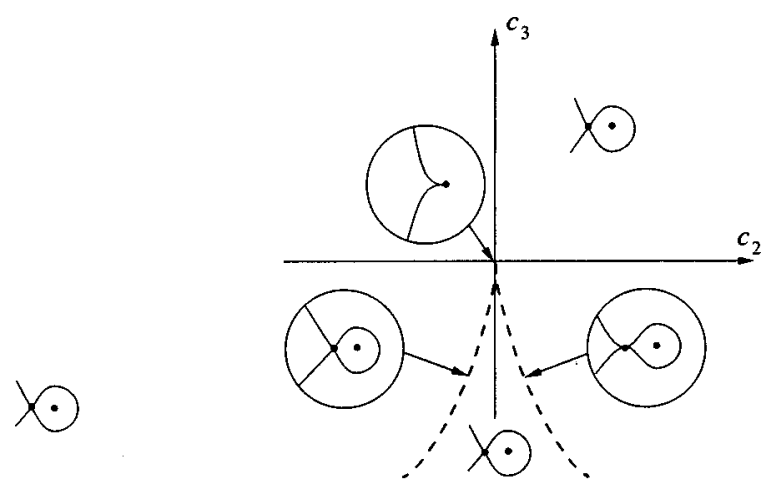

(a)

(b)

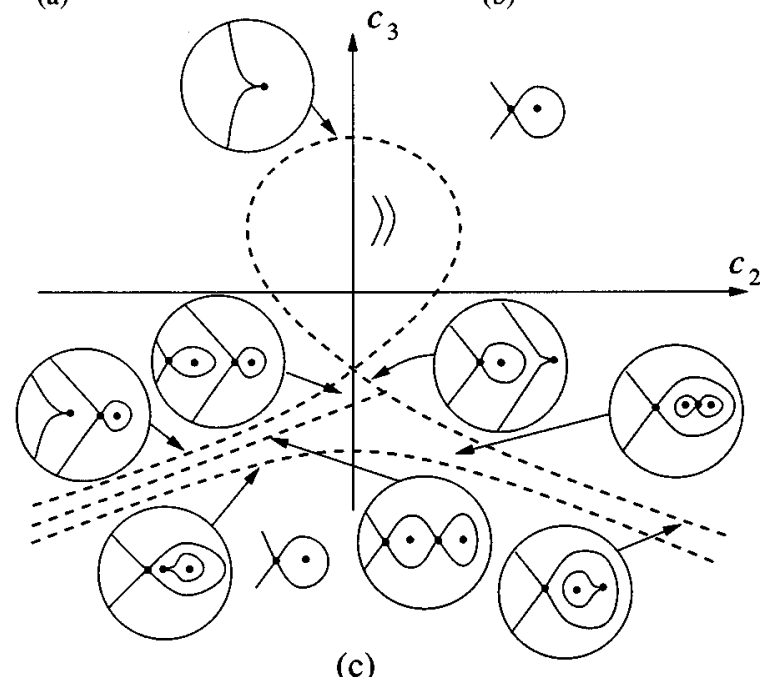

(c)

FIG. 5. Bifurcation diagram for the normal form of order 5, Eq. (43). (a) $D<0$. (b) $D=0$. (c) $D>0$.

$$
\begin{aligned}
& f^{\prime}\left(x_{1}\right)=f^{\prime}\left(x_{2}\right)=0, \\
& f^{\prime \prime}\left(x_{1}\right)<0 \wedge f^{\prime \prime}\left(x_{2}\right)<0,
\end{aligned}
$$

for $x_{1} \neq x_{2}$. By eliminating $x_{1}$ and $x_{2}$ from Eqs. (48a)-(48b) by the use of resultants, ${ }^{29}$ one finds that global bifurcations occur for

$$
\begin{aligned}
3 D & \left(9 D^{2}-66 D c_{3}^{2}+161 c_{3}^{4}+18 c_{3} c_{2}^{2}\right) \\
& =392 c_{3}^{6}+142 c_{3}^{3} c_{2}^{2}-9 c_{2}^{4} .
\end{aligned}
$$

Notice that the constraint $(48 \mathrm{c})$ is not included here and only a part of the curve (49) describe global bifurcation points. The complete bifurcation diagram is shown in Fig. 5.

\section{PATTERNS IN SYMMETRIC FLOWS}

In this section we will investigate streamline topologies under the assumption of reflectional symmetry. We take the axis of symmetry to be the $y$ axis, and the streamfunction is then an even function of $x$,

$$
\psi(-x, y)=\psi(x, y),
$$

and the expansion (2) becomes

$$
\psi=\sum_{i+j=1}^{\infty} a_{2 i, j} x^{2 i} y^{j} .
$$

The linearization of the streamline equations is now

$$
\left(\begin{array}{c}
\dot{x} \\
\dot{y}
\end{array}\right)=\left(\begin{array}{c}
a_{0,1} \\
0
\end{array}\right)+\left(\begin{array}{cc}
0 & 2 a_{0,2} \\
-2 a_{2,0} & 0
\end{array}\right)\left(\begin{array}{l}
x \\
y
\end{array}\right),
$$

so the origin is a critical point if $a_{0,1}=0$, which is a center if $|J|=4 a_{2,0} a_{0,2}$ is positive and a saddle if $|J|$ is negative. A degeneration occurs if $|J|=0$ and, as before, we consider only the case when $J$ is not the zero matrix.

We will again use normal form transformations to simplify the streamfunction at and close to the degeneracy, and the algebra proceeds as in Sec. II, with the exception that we now require that the transformations conserve the symmetry. Denoting the new coordinates $(\xi, \eta)$, this means that

$$
\xi(-x, y)=-\xi(x, y), \quad \eta(-x, y)=\eta(x, y),
$$

and it follows that the generating function (23) must be an odd function of $\xi$.

Two subcases must be distinguished. If $a_{0,2}=0$ and $a_{2,0} \neq 0(\nabla u=0, \nabla u \neq 0)$ the normal form computations lead to exactly the same result as in Theorem II.1, but with $x$ and $y$ interchanged. Thus, the symmetry that always occurs in the general normal form covers this case as well, and the assumption of symmetry give rise to no further limitations in the possible streamline patterns and their bifurcations.

In the other subcase $a_{2,0}=0$ and $a_{0,2} \neq 0 \quad(\nabla v=0, \nabla u$ $\neq 0$ ), the normal form procedure gives rise to the following.

Theorem III.1: Let $a_{0,1}, a_{2,0}$ and $\tilde{a}_{4,0}, \tilde{a}_{6,0}, \ldots$, $\tilde{a}_{2[N / 2]-2,0}$ be small parameters. Assuming the nondegeneracy conditions $a_{0,2} \neq 0, \widetilde{a}_{2[N / 2], 0} \neq 0$ a normal form of order $N$ for the streamfunction (51) is

$$
\begin{aligned}
& \psi_{N}=\frac{\sigma}{2} y^{2}+f(x), \quad f(x)=\sum_{i=1}^{[N / 2]} c_{2 i} x^{2 i}, \\
& c_{2[N / 2]}=\frac{1}{2[N / 2]},
\end{aligned}
$$

where

$$
\sigma= \begin{cases}-1, & \text { for } a_{0,2} / \widetilde{a}_{2[N / 2], 0}<0, \\ +1, & \text { for } a_{0,2} / \widetilde{a}_{2[N / 2], 0}>0,\end{cases}
$$

and $c_{2 i}, i=1, \ldots,[N / 2]$ are transformed small parameters.

In the theorem $[\cdot]$ denotes the integer part. The structure of the normal form is exactly the same as for the general case in Sec. II, only with no terms of odd order in $x$. Thus, we reach the same general conclusions here but notice that the origin is always a critical point and the total number of critical points is odd. In addition to the local bifurcation occurring when $f^{\prime \prime}(x)=0$ and $f^{\prime}(x)=0$, global bifurcation takes place when the streamfunction attains the same value at two saddle points $x_{1}, x_{2}$ that are not a symmetric pair, that is, when $\left|x_{1}\right| \neq\left|x_{2}\right|$.

The algebraic manipulations involved in obtaining the bifurcation diagrams in the symmetric case follows closely the procedure in Sec. II. We omit the details, and only show the resulting diagrams for codimension up to three in Figs. $6-9$. 
(a)
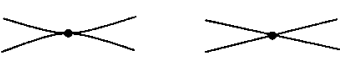

(b)
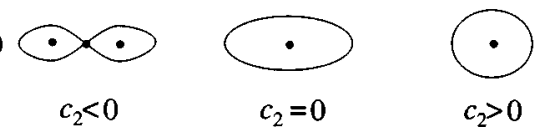

FIG. 6. Bifurcation diagram for the symmetric normal form of order 4. (a) $\sigma=-1$. (b) $\sigma=1$.

\section{THE ROLE OF THE VORTICITY TRANSPORT EQUATION IN STEADY FLOWS}

The patterns we have discussed until now are based only on the existence of a streamfunction $\psi$ for which only incompressibility is assumed. Thus, all patterns can be realized, since they can be used as initial conditions for a temporal development. Here we address the question as to whether the patterns can appear in steady flows.

For a 2-D viscous flow, the Navier-Stokes equations can be combined into the vorticity transport equation by eliminating the pressure. In a streamfunction formulation for steady flow, the equation is

$$
\nu \nabla^{4} \psi=\frac{\partial \psi}{\partial y} \frac{\partial}{\partial x}\left(\nabla^{2} \psi\right)-\frac{\partial \psi}{\partial x} \frac{\partial}{\partial y}\left(\nabla^{2} \psi\right),
$$

where $\nu$ is the kinematic viscosity. Inserting the expansion (2) and collecting terms of the same order in $x, y$ yields a series of algebraic equations for the $a_{i, j}$. The equations to order zero and one are

$$
\begin{aligned}
& x^{0} y^{0}: 8 \nu\left(a_{2,2}+3 a_{4,0}+3 a_{0,4}\right) \\
&= 2 a_{0,1}\left(3 a_{3,0}+a_{1,2}\right)-2 a_{1,0}\left(3 a_{0,3}+a_{2,1}\right), \\
& x^{1} y^{0}: 24 \nu\left(5 a_{5,0}+a_{3,2}+a_{1,4}\right) \\
&=-6 a_{1,0}\left(a_{1,3}+a_{3,1}\right)+4 a_{0,1}\left(a_{2,2}+6 a_{4,0}\right) \\
&+2 a_{1,1}\left(a_{1,2}+3 a_{3,0}\right)-4 a_{2,0}\left(a_{2,1}+3 a_{0,3}\right), \\
& x^{0} y^{1}: \quad 24 \nu\left(5 a_{0,5}+a_{2,3}+a_{4,1}\right) \\
&=6 a_{0,1}\left(a_{1,3}+a_{3,1}\right)-4 a_{0,1}\left(a_{2,2}+6 a_{0,4}\right) \\
&-2 a_{1,1}\left(a_{2,1}+3 a_{0,3}\right)+4 a_{0,2}\left(a_{1,2}+3 a_{3,0}\right) .
\end{aligned}
$$

In general, the equation associated with the term $x^{i} y^{j}$ is

$$
\begin{gathered}
\frac{(j+4) !}{j !} a_{i, j+4}+2 \frac{(i+2) !}{i !} \frac{(j+2) !}{j !} a_{i+2, j+2} \\
+\frac{(i+4) !}{i !} a_{i+4, j}=f_{i, j},
\end{gathered}
$$

where $f_{i, j}$ are nonlinear combinations of coefficients $a_{l^{\prime}, m^{\prime}}$, with $l^{\prime}+m^{\prime}<i+j+4$. The structure of these equations is clear: For a given order $k$ the set of equations for $x^{i} y^{j}$ with $i+j=k$ consists of a linear combination of $a_{l, m}$, with $l$ $+m=k+4$ on the left hand side and a nonlinear combination of $a_{l^{\prime}, m^{\prime}}$ with $l^{\prime}+m^{\prime}<k+4$ on the right-hand side. Hence, these equations give linear constraints on the coefficients of a certain order expressed in terms of lower-order coefficients.

There are $k+1$ equations in the set to order $k$ but $k+5$ unknown coefficients of order $k+4$. Thus, the system is not fully determined. However, it is easy to see that the system has rank $k+1$ and that $a_{k, 0}$ can be chosen as one of the four free parameters. Hence the equations give no information about $a_{k, 0}$ which enters linearly in the decisive parameter $\widetilde{a}_{k, 0}$ defined in Eqs. (19), which consequently is left undetermined.

Furthermore, there is no information about coefficients of order less than four, so we conclude that the vorticity transport equation does not give any constraints on steady streamline patterns and their bifurcations.

In the limit $\nu \rightarrow \infty$ we have Stokes flow, and Eq. (56) is replaced by

$$
\nabla^{4} \psi=0
$$

and the right hand side of Eq. (58) becomes zero. Since the arguments above were based entirely on the linear part of the Navier-Stokes equations we reach the same conclusion for Stokes flow: All topologies and bifurcations described in Sec. II can occur in steady Stokes flow.

Steady flow was also considered by Bakker $^{23}$ in his analysis of flow close to a wall. However, since the constraints from the Navier-Stokes equations were inserted before the topological analysis, their role was not clear. In an analysis similar to the present one, Hartnack ${ }^{25}$ found that

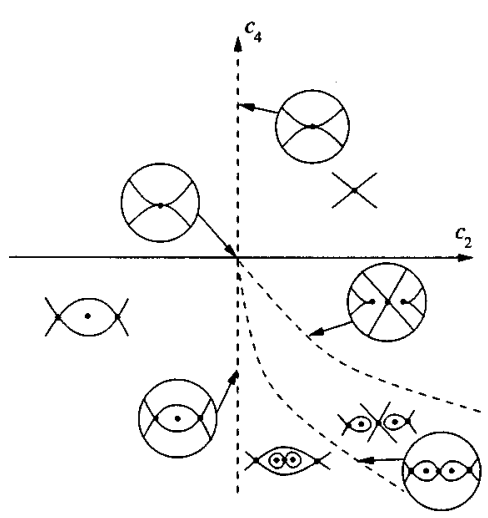

(a)

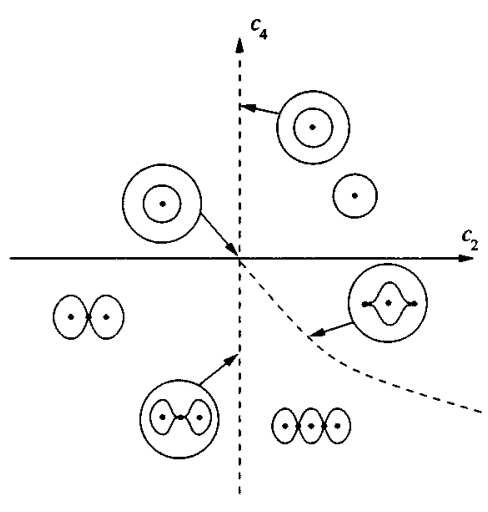

(b)

FIG. 7. Bifurcation diagram for the symmetric normal form of order 6. (a) $\sigma=-1$. (b) $\sigma=1$. 


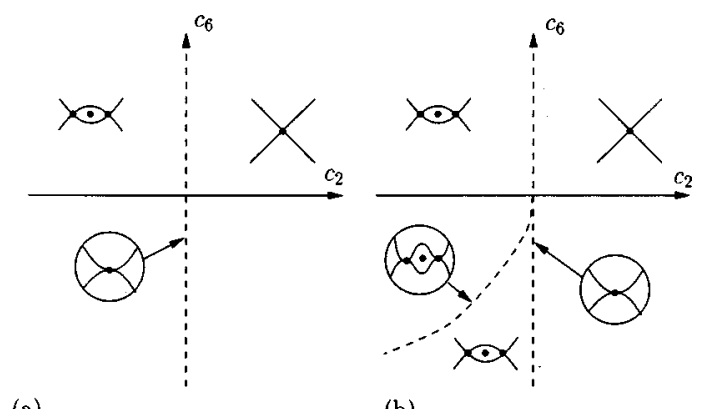

(a)
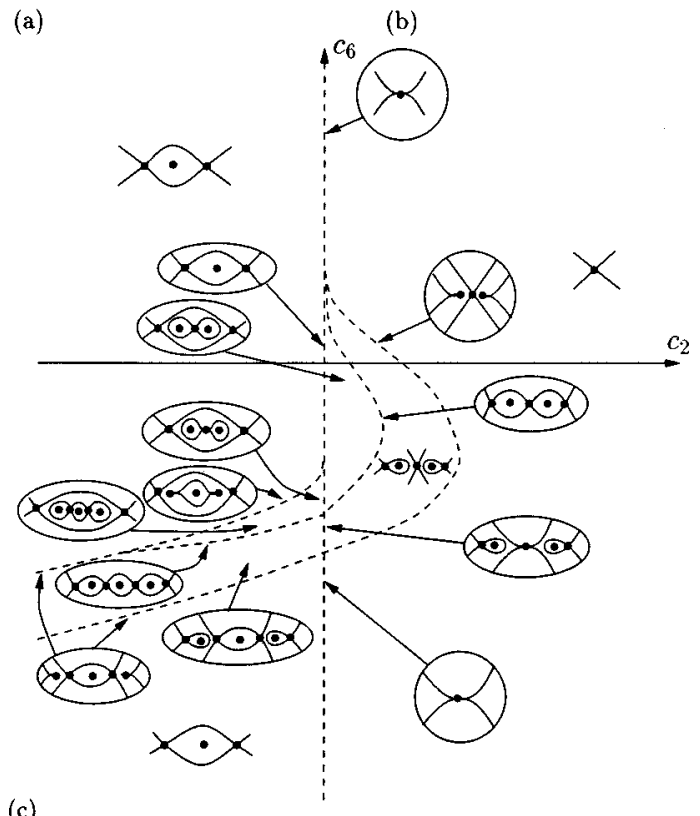

(c)

FIG. 8. Bifurcation diagram for the symmetric normal form of order 8 with $\sigma=1$. (a) $D<0$. (b) $D=0$. (c) $D>0$. Here, $D=16 c_{6}^{2}-\frac{16}{3} c_{4}$.

steadiness poses no topological constraints for the unfolding of a simple degeneracy at a wall. However, in a low-order case with a nonsimple degeneracy some patterns were ruled out for steady flow.

For flows away from walls, a systematic study of the role of steadiness for nonsimple degeneracies has not been performed. However, from the vorticity transport equation (56) it follows that only coefficients to terms of fourth order or higher in the streamfunction are constrained. Further nonsimple degeneracies with topologies determined by fourthorder terms or higher in the streamfunction are of codimension 4 or more. This means that such degeneracies are not very common in flows, and the question of whether steadiness imposes a restriction on the topology of these degeneracies or not becomes rather academic.

Let us now consider the more subtle case of flows with reflectional symmetry. Without loss of generality the symmetry line will be taken as $x=0$, and the streamfunction now has the expansion (51), all terms being even in $x$. Equations (57) now become

$$
\begin{array}{ll}
x^{0} y^{0}: & a_{2,2}+3 a_{4,0}+3 a_{0,4}=0, \\
x^{1} y^{0}: & 0=2 a_{0,1}\left(a_{2,2}+6 a_{4,0}\right)-2 a_{2,0}\left(a_{2,1}+3 a_{0,3}\right), \\
x^{0} y^{1}: & 5 a_{0,5}+a_{2,3}+a_{4,1}=0 .
\end{array}
$$

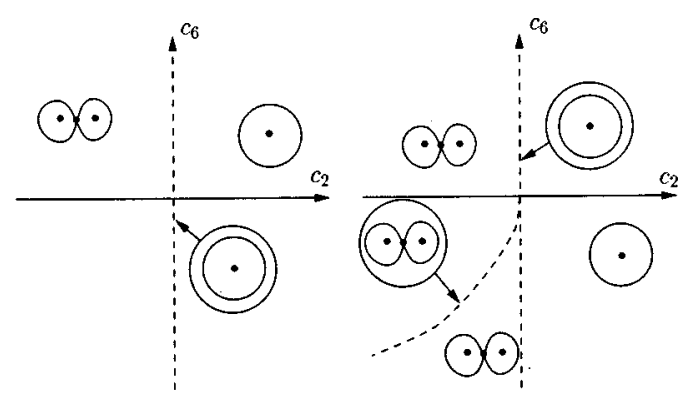

(a)

(b)

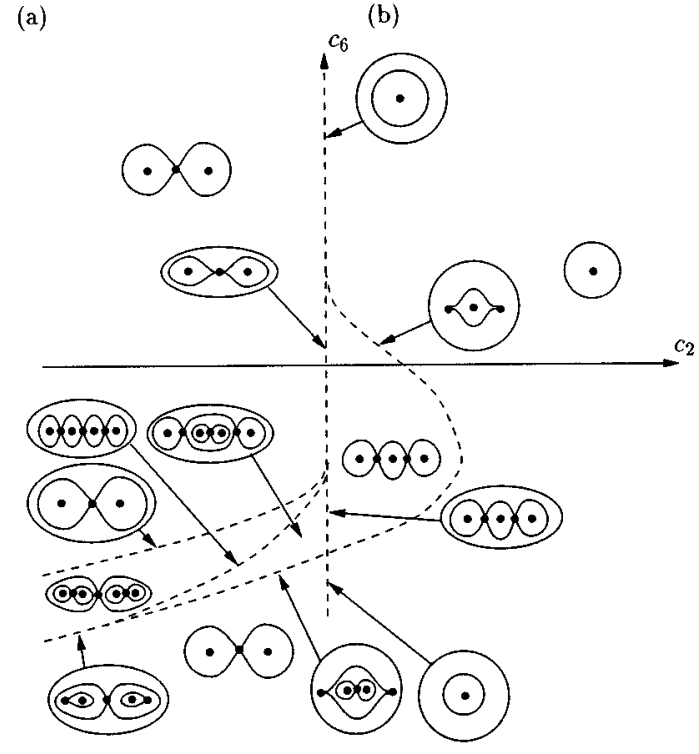

(c)

FIG. 9. Bifurcation diagram for the symmetric normal form of order 8 with $\sigma=-1$. (a) $D<0$. (b) $D=0$. (c) $D>0$.

A problem for the two equations of order $k=1$ for which the unknowns are the coefficients of order five is evident. Solutions exist only if the nonlinear constraint, Eq. (60b), is fulfilled. Furthermore, if we continue to the equations for higher orders $k$ we encounter a similar problem for each equation with an odd power of $x$. This gives rise to infinitely many nonlinear constraints that involve low-order terms in a very complicated pattern.

We leave the question on the solution of these constraints unresolved here, and turn to the case of Stokes flow with symmetry. Again we get some equations with zero lefthand sides, but now the right-hand sides are all zero, and we are back to a structure similar to the unsymmetric case. The nonzero equations of order $k$ constitute an underdetermined homogeneous linear system, and only the coefficients of even order have topological interest according to Theorem III.1. It is not difficult to see that the $a_{2 n, 0}$ can be chosen as free parameters, and again the $\widetilde{a}_{2 n, 0}$ are undetermined.

Thus, for symmetric Stokes flow steadiness poses no topological constraint. Whether it distinguishes Stokes flow from Navier-Stokes flow is an open problem.

\section{APPLICATIONS}

\section{A. Stokes flow in a rectangular cavity}

As an application of the flow patterns found, we investigate Stokes flow in a rectangular cavity with length $a$ and 


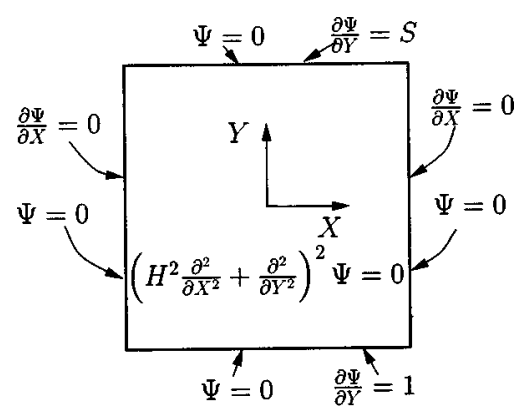

FIG. 10. The geometry of Stokes flow in a rectangular cavity.

height $b$. The flow is driven by sliding the top and the bottom of the box. These two boundaries are allowed to move independently of each other at constant speed $u_{t}$ and $u_{b}$, respectively. The two sidewalls are fixed. The flow is governed by the Stokes equation,

$$
\nabla^{4} \psi=0
$$

The axes of the system and the streamfunction are nondimensionalized through

$$
x=a X, \quad y=b Y, \quad \Psi=\frac{\psi}{b u_{b}},
$$

which applied to Eq. (61) gives the nondimensional equation

$$
H^{4} \frac{\partial^{4} \Psi}{\partial X^{4}}+2 H^{2} \frac{\partial^{4} \Psi}{\partial X^{2} \partial Y^{2}}+\frac{\partial^{4} \Psi}{\partial Y^{4}}=0, \quad \text { with } H=\frac{b}{a},
$$

which has to be satisfied in a unit square. The situation is sketched in Fig. 10.

With the given geometry there are two parameters which can be varied independently. The height to length ratio,

$$
H=\frac{b}{a},
$$

and the ratio of the top and bottom wall velocities,

$$
S=\frac{u_{t}}{u_{b}} .
$$

By varying these two one obtains different flow topologies.

The sketched cavity flow has been studied recently numerically by Kelmanson and Lonsdale. ${ }^{26}$ They solved the problem using a numerical integral equation method. Shankar ${ }^{30}$ also studied the cavity flow, though he considered only the motion of one endwall and used a numerical method using a truncated eigenfunction expansion. Here we show solutions obtained by a finite difference scheme developed by Altas et al. ${ }^{31}$ This scheme uses not only the streamfunction as unknown but also the first derivatives. This expands the number of unknowns to three. This increase in unknowns is compensated by the exact implementation of the boundary conditions instead of introducing approximations at boundaries. Also, this scheme gives direct access to the critical points since these can be found as intersections of the isoclines $\psi_{X}=0$ and $\psi_{Y}=0$.

Since the system has two independent parameters, one should not expect to observe degenerate streamline configu-

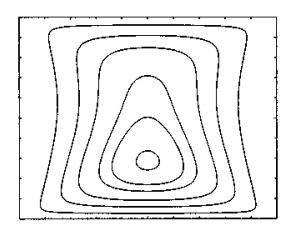

(a)

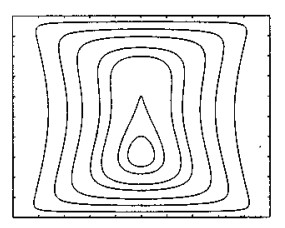

(b)

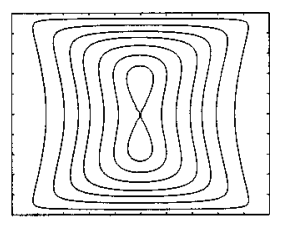

(c)

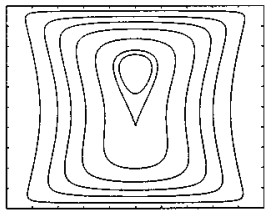

(d)

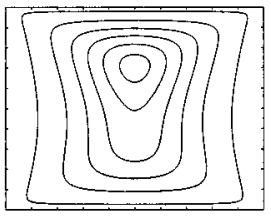

(e)
FIG. 11. Streamline patterns for Stokes flow in double lid driven cavity, for different values of the velocity ratio $S$ : (a) $S=-0.70$; (b) $S=-0.90$; (c) $S=-1.00$, (d) $S=-1.10$; and (e) $S=-1.30$.

rations of codimension more than 2 . Using a coordinate system fixed at the center of the box (as shown in Fig. 10) the system has reflectional symmetry in the $Y$ axis and the domain of the problem is bounded giving closed streamlines. The bifurcation diagrams that are appropriate for this system are consequently Fig. 4(a) (with $x$ and $y$ interchanged), Fig. 6, and Fig. 7(b).

In Fig. 11 a series of streamline patterns is displayed for different values of the velocity ratio $S$ and a fixed height to length ratio of unity. By variation of the velocity ratio, one encounters exactly the bifurcations of Fig. 4(a). In Fig. 11(a) only one center point is present, by decreasing $S$ to around -0.90 a cusp critical point appears above the center point. By decreasing $S$ below -0.90 this cusp critical point evolves into a saddle center configuration in (c), giving one large vortex with an inner "figure-eight"-shaped structure. Through a further decrease in $S$ the top vortex grows in strength, annihilating the bottom vortex through a second cusp bifurcation at $S=-1.10$. The process thus observed corresponds to varying $c_{1}$ across the cusp-shaped bifurcation curve along a curve with $c_{2}<0$ in Fig. 4(a).

If this experiment was repeated at a slightly different value of $H$, the same behavior is expected, but with the specific values of $S$, where the bifurcations occur also slightly altered, corresponding to moving along a slightly different curve in the $\left(c_{1}, c_{2}\right)$ parameter space. Hence, by varying the two physical parameters $H, S$, a bifurcation diagram corresponding to Fig. 4 will result. It may be deformed but the cusp shape will persist.

Other bifurcations are present in the system. Again, for $H=1$ Kelmanson and Lonsdale ${ }^{26}$ observe in the range $0.05<S<0.25$ the metamorphosis of a "double eddy" (that is, a "figure-eight") into a "treble eddy" and further into a single eddy. The patterns are shown in their Figs. 5, 6. This sequence corresponds exactly to the bifurcations in Fig. 7(b) when $c_{2}$ is changed from negative to positive along a curve with $c_{4}<0$, crossing both bifurcation curves below the $c_{2}$ axis. We conclude that the complete bifurcation diagram of Fig. 7(b) is expected to be present in a $H, S$ bifurcation diagram. 
(a)

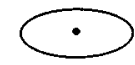

(f)

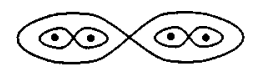

(b)

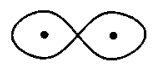

(c)
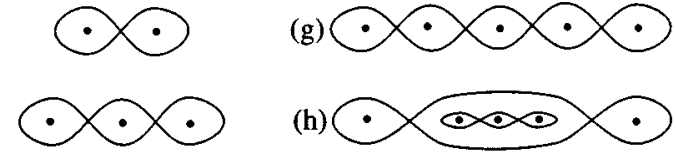

(d)

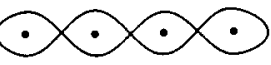

(i)

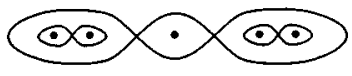

(e)

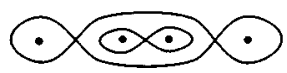

FIG. 12. The flow structures listed by Gaskell et al. (Ref. 27) as being possible in a Stokes flow in a cavity.

Hence, the present theory can guide the construction of pattern bifurcation diagrams. This approach has been used by applying the corresponding bifurcation diagrams for axisymmetric flow $^{18}$ to map the topologies in the steady vortex breakdown. ${ }^{19,32}$

\section{B. Stokes flow between concentric cylinders}

Gaskell et al. ${ }^{27}$ have considered Stokes flow in a halffilled annular region between rotating concentric cylinders. The problem is conveniently described in polar coordinates, where the lines with $\theta= \pm \pi / 2$ are the free surfaces. The resulting boundary value problem is characterized by two dimensionless parameters: the ratio $\bar{R}$ of the radii of the cylinders and the ratio $S$ of the peripheral speeds of the cylinders. A series solution is obtained assuming reflectional symmetry across the centerline $\theta=0$, and a rich set of eddying motions is found by evaluating the solution numerically.

A basic flow in the system consists of a vortex with its center on the centerline. As the parameters are varied $(\bar{R}$ being the most important), this can bifurcate to a saddle and two off-axis centers, like in Fig. 6(b) for decreasing values of $c_{2}$. A further variation of parameters can change the saddle back to a center and at the same time create two off-axis saddles, as in Fig. 6(a) for decreasing values of $c_{2}$. These two bifurcations occur repeatedly, resulting in more and more complex patterns as the number of critical points rise.

Gaskell et al. list a number of flow structures (reproduced in Fig. 12) that they consider to be possible for the flow, though only the patterns (a), (b), (c), and (h) were realized.

From the present analysis it is clear that the patterns (d) and (g) are not to be expected numerically. They are structurally unstable due to the multiple saddle connections and a slight change of parameters can break some of the connections. For example, pattern (d) occurs in Fig. 9(c), but along a bifurcation surface only.

The lack of patterns (f), (i) is more surprising. Since there are two control parameters in the system, one would expect bifurcations with codimension up to two to occur generically. Such bifurcations are exactly those described in Fig. 7, and if the "unfinished" separatrices in Fig. 7(a) are joined around far off-axis centers, one finds indeed that pat-

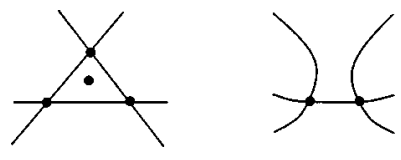

FIG. 13. Flow patterns that do not appear in the unfoldings of a simple linear degenerate critical point: Critical points in a triangle and saddles with a single heteroclinic connection.

tern (f) can bifurcate from pattern (b) through the creation of off-axis cusp points.

An explanation for the missing bifurcation of critical points off the axis may be found in the shape of the flow domain. The existence of the series solution hinges on the free surfaces being radial lines. Special topological properties may be associated with these solutions. These can be destroyed while keeping the reflectional symmetry, for instance, by changing the amount of fluid such that the annulus is not exactly half-filled. Hence, if the height of the free surface is varied as a third parameter, it may well be possible to encounter a more complex set of flow topologies and bifurcations, and the subset found in Ref. 27 may turn out to be quite special.

\section{CONCLUSIONS}

The use of normal forms in the analysis of flow patterns gives a significant simplification and allows an easy determination of the possible degenerate patterns and their bifurcations, even to quite high codimension. The structure of the normal form depends only on the Jacobian matrix of the velocity field at a degenerate critical point. In the case considered here, with a simple linear degeneracy, all critical points lie on a line in the normal form coordinates, and configurations with critical points in a triangle cannot appear. Also, a single heteroclinic connection between saddles violates the inherent symmetry of the normal forms. See Fig. 13. All vortices also have the same sign and an interaction between opposite vortices as shown in Fig. 14 is not captured.

Hence, an understanding of a number of interesting and important patterns require an analysis of degeneracies with a zero Jacobian matrix. Unfortunately, a direct use of the normal form approach in this case gives no simplifications at all. A systematic elimination of unimportant terms is difficult, reflecting the much richer set of structures that is possible here. In a similar situation for the flow close to a wall, Hartnack ${ }^{25}$ have obtained simplification of low-order terms. The same approach may be used for the present case.

The topological classifications obtained in this paper hinge on the coefficients of the normal forms. In principle, these can be expressed in terms of the Taylor expansion coefficients $a_{i, j}$ of the streamfunction, which, in turn, can be interpreted physically in terms of the stress tensor, as indi-

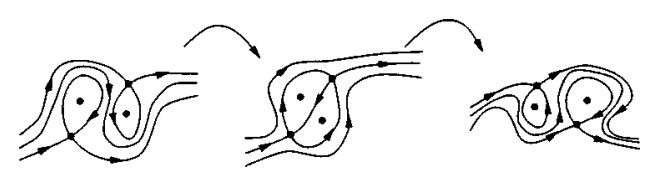

FIG. 14. The interaction of two vortices with opposite rotation. 
cated in Sec. II A. However, apart from the coefficients of the very lowest orders, this algebraic connection is a very complicated one that we have not even attempted to write out explicitly. This is not a flaw of the approach used here but a basic property of fluid patterns. In consequence, there are, in general, no simple physical quantities that can be used as indicators for pattern bifurcations. Thus, it is inherently difficult to single out the physical mechanisms that are responsible for pattern changes, except in the simplest cases.

While a topological classification in itself cannot solve a problem in fluid mechanics, it provides guidelines for identification of structures in any flow. We have indicated how this could be done for two cases of Stokes flow. A more detailed study of the structures in the Stokes flow in a cavity is in preparation.

${ }^{1}$ H. J. Lugt, “The dilemma of defining a vortex," in Recent Developments in Theoretical and Experimental Fluid Mechanics (Springer-Verlag, Berlin, 1979), pp. 309-321.

${ }^{2}$ M. Tobak and D. J. Peake, "Topology of three-dimensional separated flows," Annu. Rev. Fluid Mech. 14, 61 (1982).

${ }^{3}$ A. E. Perry and M. S. Chong, "A description of eddying motions and flow patterns using critical-point concepts," Annu. Rev. Fluid Mech. 19, 125 (1987)

${ }^{4}$ R. Legendre, "Séparation de l'écoulement laminaire tridimensionel," Rech. Aerosp. 54, 3 (1956).

${ }^{5} \mathrm{~K}$. Oswatitsch, "Die ablösungsbedingung von grenzschichten," in IUTAM Symposium on Boundary Layer Research (Springer-Verlag, Berlin, 1958), pp. $357-364$.

${ }^{6}$ A. Davey, "Boundary-layer flow at a saddle point of attachment," J. Fluid Mech. 10, 593 (1961).

${ }^{7}$ M. J. Lighthill, "Attachment and separation in three-dimensional flow," in, Laminar Boundary Layers, edited by L. Rosenhead (Clarendon, Oxford, 1963), p. 72.

${ }^{8}$ J. C. R. Hunt, C. J. Abell, J. A. Peterka, and H. Woo, “Kinematical studies of the flow around free or surface-mounted obstacles; applying topology to flow visualization,'’ J. Fluid Mech. 86, 179 (1978).

${ }^{9}$ U. Dallmann, "Topological structures of three-dimensional flow separations," IB 221-82 A 07, DFVLR, 1983.

${ }^{10}$ U. Dallmann, “Three-dimensional vortex structures and vorticity topology,' Fluid Dyn. Res. 3, 183 (1988).

${ }^{11}$ U. Dallmann and H. Gebing, "Flow attachment at flow separation lines," Acta Mech. 4, 47 (1994).

${ }^{12}$ A. E. Perry, M. S. Chong, and T. T. Lim, "The vortex-shedding process behind two-dimensional bluff bodies," J. Fluid Mech. 116, 77 (1982).

${ }^{13}$ A. E. Perry and T. R. Steiner, "Large-scale vortex structures in turbulent wakes behind bluff bodies. Part 1. vortex formation processes,' J. Fluid Mech. 174, 233 (1987).

${ }^{14}$ K. Shariff, T. H. Pulliam, and J. M. Ottino, "A dynamical systems analysis of kinematics in the time-periodic wake of a circular cylinder," in Vortex dynamics and vortex methods (Seattle, WA, 1990), Lectures in Applied Mathematics (American Mathematical Society, Providence, RI, 1991), Vol. 28, pp. 613-646.

${ }^{15}$ H. J. Lugt, "Local properties at a viscous free surface," Phys. Fluids 30, 3647 (1987)

${ }^{16} \mathrm{M}$. Brøns, "Topological fluid dynamics of interfacial flows,"' Phys. Fluids 6, 2730 (1994).

${ }^{17}$ T. P. Chiang, T. W. H. Sheu, and S. F. Tsai, "Topological flow structures in backward-facing step channels," Comput. Fluids 26, 321 (1997).

${ }^{18} \mathrm{M}$. Brøns, "Topological fluid mechanics of axisymmetric flows," in Simulation and Identification of Organized Structures in Flows, edited by J. N. Sørensen, E. J. Hopfinger, and N. Aubrey (Klüwer Academic, Dordrecht, 1998), pp. 213-222.

${ }^{19}$ M. Brøns, L. K. Voigt, and J. N. Sørensen, "Streamline topology of steady axisymmetric vortex breakdown in a cylinder with co- and counterrotating end-covers," DCAMM Report 582, Technical University of Denmark, June 1998.

${ }^{20} \mathrm{H}$. Aref and M. Brøns, "On stagnation points and streamline topology in vortex flows,"' J. Fluid Mech. 370, 1 (1998).

${ }^{21} \mathrm{R}$. M. Ford, "Critical point detection in fluid flow images using dynamical system properties,' Pattern Recogn. 30, 1991 (1997).

${ }^{22} \mathrm{P}$. Vorobieff and D. Rockwell, "Wavelet filtering for topological decomposition of flows." Int. J. Imaging Syst. Technol. 7, 211 (1996).

${ }^{23}$ P. G. Bakker, Bifurcations in Flow Patterns (Klüwer Academic, 1991).

${ }^{24} \mathrm{~S}$. Wiggins, "Introduction to applied nonlinear dynamical systems and chaos," Texts in Applied Mathematics (Springer-Verlag, New York, 1990), Vol. 2.

${ }^{25}$ J. N. Hartnack, "Streamline topologies near a fixed wall using normal forms," DCAMM Report 581, Technical University of Denmark, 1998, to appear in Acta Mech.

${ }^{26} \mathrm{M}$. A. Kelmanson and B. Lonsdale, "Eddy genesis in the double-liddriven cavity,”' Q. J. Mech. Appl. Math. 49, 635 (1996).

${ }^{27}$ P. H. Gaskell, M. D. Savage, and M. Wilson, "Stokes flow in a half-filled annulus between rotating coaxial cylinders," J. Fluid Mech. 337, 263 (1997).

${ }^{28}$ H. Goldstein, Classical Mechanics (Addison-Wesley, Reading MA, 1950).

${ }^{29}$ B. L. van der Waerden, Algebra (Springer-Verlag, Berlin, 1966).

${ }^{30} \mathrm{P}$. N. Shankar, "The eddy structure in Stokes flow in a cavity," J. Fluid Mech. 250, 371 (1993).

${ }^{31}$ I. Altas, J. Dym, M. Gupta, and R. Manohar, "Multigrid solution of automatically generated high order discretizations for the biharmonic equation," SIAM J. Sci. Comput. 19, 1575 (1998).

${ }^{32}$ J. F. Jørgensen and J. C. M. Troest, “Topological and numerical studies of vortex structures in two-dimensional flow in an open cylindrical container with rotating bottom,' Master's thesis, Department of Mathematics, Technical University of Denmark, 1998. 